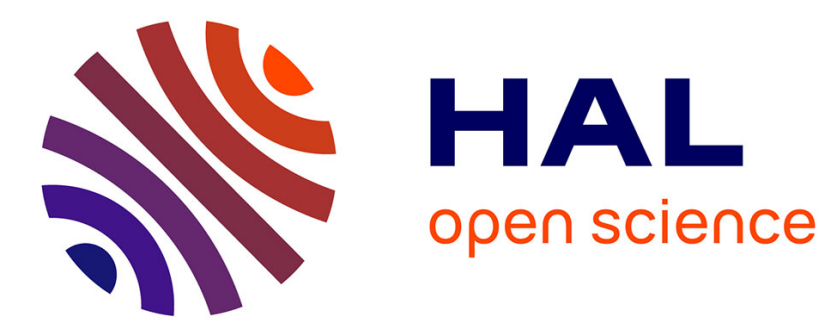

\title{
Rapid, High-Level Performance Estimation for DSE Using Calibrated Weight Tables
}

Kasra Moazzemi, Smit Patel, Shen Feng, Gunar Schirner

\section{To cite this version:}

Kasra Moazzemi, Smit Patel, Shen Feng, Gunar Schirner. Rapid, High-Level Performance Estimation for DSE Using Calibrated Weight Tables. 5th International Embedded Systems Symposium (IESS), Nov 2015, Foz do Iguaçu, Brazil. pp.197-209, 10.1007/978-3-319-90023-0_16 . hal-01854171

\section{HAL Id: hal-01854171 \\ https://hal.inria.fr/hal-01854171}

Submitted on 6 Aug 2018

HAL is a multi-disciplinary open access archive for the deposit and dissemination of scientific research documents, whether they are published or not. The documents may come from teaching and research institutions in France or abroad, or from public or private research centers.
L'archive ouverte pluridisciplinaire HAL, est destinée au dépôt et à la diffusion de documents scientifiques de niveau recherche, publiés ou non, émanant des établissements d'enseignement et de recherche français ou étrangers, des laboratoires publics ou privés. 


\title{
Rapid, High-Level Performance Estimation for DSE using Calibrated Weight Tables
}

\author{
Kasra Moazzemi, Smit Patel, Shen Feng, and Gunar Schirner \\ moazzemi.k@husky.neu.edu, patel.smi@husky.neu.edu, \\ schirner@ece.neu.edu \\ Department of Electrical and Computer Engineering, \\ Northeastern University, Boston, MA, USA
}

\begin{abstract}
Automated Design Space Exploration (DSE) is a critical part of system-level design. It relies on performance estimation to evaluate design alternatives. However, since a plethora of design alternatives need to be compared, the run-time of performance estimation itself may pose a bottleneck. In DSE, fastest performance estimation is of essence while some accuracy may be sacrificed. Fast estimation can be realised through capturing application demand, as well as Processing Element (PE) supply (later on called weight table) in a matrix each. Then, performance estimation (retargeting) is reduced to a matrix multiplication. However, defining the weight table from a data sheet is impracticle due to the multitude of (micro-) architecture aspects.

This paper introduces a novel methodology, WeiCal, for automatically generating Weight Tables in the context of $\mathrm{C}$ source-level estimation using application profiling and Linear Programming (LP). LP solving is based on the measured performance of training benchmarks on an actual PE. We validated WeiCal using a synthetic processor and benchmark model, and also analyse the impact of non-observable features on estimation accuracy. We evaluate the efficiency using 49 benchmarks on 2 different processors with varying configurations (multiple memory configurations and software optimizations). On a $3.1 \mathrm{GHz}$ i5-3450 Intel host, 25 million estimations / second can be obtained regardless of the application size and PE complexity. The accuracy is sufficient for early DSE with a $24 \%$ average error.
\end{abstract}

\section{Introduction}

Recent advances in technology have expanded the design options in terms of number and type of processors as well as their configurations such as interconnects and memory hierarchy. When this flexibility of design is coupled with the increasing pressure of time to market, performance exploration of the design space becomes exponentially difficult. Current approaches try to automate the Design Space Exploration (DSE). In any DSE, two questions need to be addressed. One is how to traverse the design space and other is how to assess the fitness of each design instance - all unique combinations of platforms and mappings. Millions of design options will be traversed before making a design 
decision. Evaluating the fitness of each design option falls on the time critical path of DSE and is at most importance here. Simulation based approaches can be highly accurate but too slow for DSE. New approaches are needed for rapid high-level performance estimation in context of DSE.

This paper revisits the retargetable profiling for rapid, early system-level design space exploration, introduced in [1] and improves upon it. The retargatable profiler [1] uses a weight table, which is a matrix of Processing Elements (PE) performance cost (cycles) of each high-level operation for all data types. One of the main challenges of retargetable profiling is that the weight tables need to be manually defined. The accuracy of weight table impacts the accuracy of final estimation. Due to manual extraction of these weight tables from the data sheet, this process is time consuming and error prone. Moreover, because of the high-level abstraction, only a few features of the processor are observable (can be quantified). For example, $\mathrm{C}$ statements do not reveal from where an operand needs to be fetched from within the memory hierarchy. Therefore, the cycles captured in the weight tables have to statistically include these non-observable characteristics such as memory accesses and pipeline stalls. These elements which can affect the performance but are not observed during execution make it very difficult to manually populate the weight table. In this paper, we present a methodology and a framework Weight Calibration (WeiCal) to automatically populate more realistic weight tables paving the way to efficient DSE.

The WeiCal framework consists of Calibration and Retargeting. A set of training benchmarks are profiled along-with the actual execution of those benchmarks on the target PE in calibration phase, generating a Linear Program (LP). This is fed to an LP Solver which defines the weight table of the particular PE, implicitly considering the vast number of architectural and micro architectural features. To estimate the performance of a target application, in the retargeting phase, it is profiled once to extract computational demand. Then, performance is estimated purely through a static approach by a simple matrix multiplication of the PE weight table and the application's computational demand. With this, multiple iterations of the retargeting step can rapidly estimate the performance of different target processors. The advantage of this method is that the application is simulated only once (for profiling), avoiding long repetitive simulations. In addition, due to fast computation in retargeting stage, this approach is particularly suitable for rapid comparisons in early DSE.

We validate WeiCal using a synthetic processor and benchmark model, and also analyse the limitations of this approach. We evaluate the efficiency of the proposed methodology using 49 benchmarks on 2 different processors with varying configurations (multiple memory configurations and software optimizations). On a $3.1 \mathrm{GHz}$ i5-3450 Intel host, 25 million estimations / second can be obtained regardless of the application size and PE complexity. The accuracy is sufficient for early DSE with a $24 \%$ average error.

The rest of this paper is organised as follows: Section 2 presents an overview of work related to this approach. Section 3 introduces retargetable profiling. Section 4 presents WeiCal and 5 presents implementation. Section 6 presents a 
synthetic model to validate the approach. Section 7 shows experimental results and Section 8 concludes the paper.

\section{Related Work}

Many estimation methods have been proposed trying to solve different challenges in estimation such as accuracy, speed and being application specific. They generally estimate based on one of three abstraction levels: : source-level (high-level), intermediate-level and binary-level (low-level). At high-level, fewer details are taken into account. It is faster and retargetable but less accurate in terms of absolute performance numbers. On the other side, low-level estimation benefits from more target architecture knowledge increasing accuracy at cost of simulation speed. While low-level may produce cycle approximate estimations for detailed analysis, high-level estimation is more suitable for DSE due to estimation speed.

Various high-level estimation techniques have been proposed. The authors of [2] propose an approach which has limitations due to compiler optimizations. Wang et al. [3] present an approach which takes compiler optimizations into account. However, both these approaches rely on simulation for estimation. The authors of [4] propose a compiler-assisted technique to rapidly estimate without simulation. However, this approach is developed for the FPGA based processors. Oyamada et al.[5] present an integrated approach for system design and performance analysis. An analytic approach based on neural networks is used for high-level software performance estimation. This approach takes about 17 seconds to estimate the performance of an MPEG4 encoder application. A hybrid simulation method is introduced in [6] which also uses a cache simulator to measure memory access delay. [9] presents a complementary method for increasing the accuracy of approaches that are annotating timing information into source code by mapping binary representation to source level. This approach requires the source code and the binary-level CFG. In [7], an estimation approach is proposed for transaction level. One drawback of this work is that the mapping between the $\mathrm{C}$ processes to PEs should be determined before using this estimation approach. These approaches are suitable for estimating the performance of a PE, but efficient design space exploration requires faster retargetability.

Javaid et al. [8] propose two estimation mechanisms whose goal is to minimise the estimation time. Though the approach is retargetable for pipelined MPSoCs, the performance estimation of individual component of design space is yet not retargetable. Mohanty et al. [10] present a mechanism for DSE using interpretive simulation which requires specific inputs to the proposed model.

We base our work on [1] for DSE as it is retargetable and does not involve simulating the target application across the design space. However, the estimation accuracy of this approach is largely dependent on the weight table entries of PEs in the design space.

\section{Retargetable Profiling}

Retargetable profiling [1] is a high-level estimation technique, which is divided into two stages - Profiling and Retargeting. 
In the profiling stage, the system specification is instrumented and simulated to gather basic block execution counts. Static analysis then computes the number of operations executed (distinguished by type) for each data-type executed and stored in the form of specification characteristic table. This specification characteristic table has the format same as weight table of a PE. Note, the profiling stage is done only once per application.

In the Retargeting stage, the designer decides the mapping of behaviour to a PE. Performance of executing the behavior on the selected PE is estimated by multiplying specification characteristics (obtained from profiling) with delay values stored in the weight table of that $\mathrm{PE}$. The total performance $(\mathrm{E})$ of that $\mathrm{PE}$ is computed through a matrix multiplication and sum.

$$
\left.E=\sum_{\text {OpType DataType }} \sum_{\text {DopType.DataType }} \times W_{\text {OpType.DataType }}\right)
$$

where $W_{\text {OpType.DataType }}$ is the weight (i.e. clock cycles) and $F_{\text {OpType.DataType }}$ is the occurrence frequency of each operation type OpType of data-type DataType. Since retargeting consists of a pure static approach, it avoids the time-consuming steps of simulation and profiling.

With its extremely fast estimation speed, retargetable profiling is very suitable for DSE. However, it requires a tedious manual step of extracting the weight table information (execution delay for each operation and datatype combination) from the data sheet of each PE. Considering that the IP vendors have their unique way of representing this information, data collection can be timeconsuming. Furthermore, dedicating only one table for each processor limits the designer to one configuration in terms of compiler optimizations and hardware configurations. This limitation makes the design space too simplistic. Moreover, some affecting elements are unknown, such as details of pipeline and data forwarding, because the vendors often do not release this information. This poses many challenges to manually define weight tables. This paper, introduces a framework for automatically generating the weight tables by calibration.

\section{Weight Calibration (WeiCal)}

This section proposes a technique for calibrating PE weight tables. It automatically populates the weight tables using a training set of benchmarks and a Linear Programming Formulation (LPF). This methodology expands flexibility of the retargetable profiling approach [1], and can increase the accuracy by implicitly considering more architectural features.

\subsection{An Overview of the Framework}

As shown in Figure 1, WeiCal generates weight tables for PEs according to a set of training benchmarks. Every benchmark is captured in SpecC language (based on ANSI-C) and then profiled with SCProf profiler [1] to determine the application computation demand. It includes the frequency of all operation types for each data type for the whole application. Each benchmark is also executed on a real processor (Processing Element $(\mathrm{PE})$ ) to obtain accurate benchmark 
execution cycles. An LP formulation is constructed using the benchmark characteristics and measured execution cycles. Solving this linear system yields the weight table for the PE.

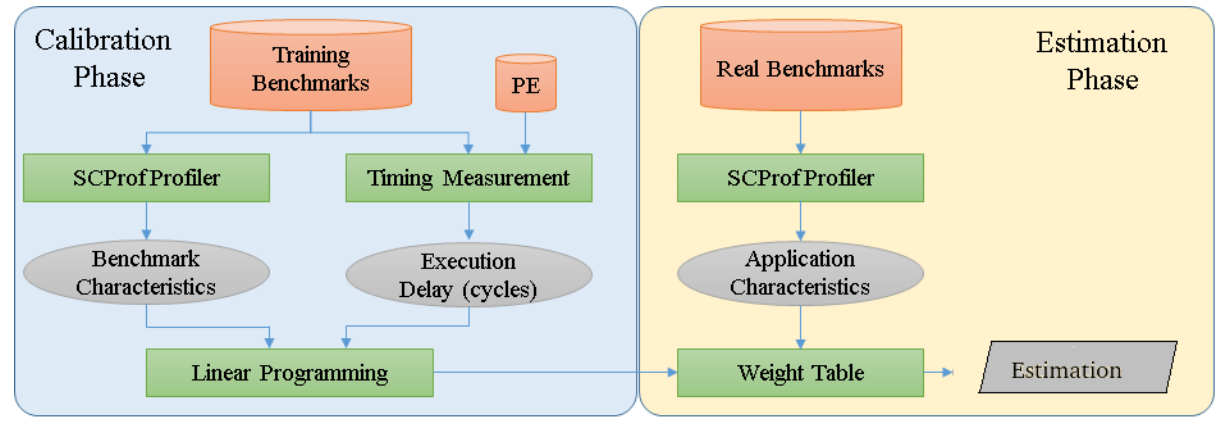

Fig. 1. Framework Flow

\subsection{Linear Programming Formulation (LPF)}

This section describes the LPF to obtain weight tables. For each benchmark $i$ on a particular PE, $\left(B_{i}\right)$ specifies the measured execution time (cycles). $D_{i j}$ denotes the computational demand of benchmark $i$ as determined by profiler. For ease of explanation, we fold every combination of operation-type and data-type into one index $j$. $W_{j}$ denotes the weight of one operation and datatype combination. An equation is generated for each benchmark which includes the weights of each operation-data-type combination, occurrence frequency of each combination and total execution time. The number of equations would be equal to the number of benchmarks available. The weights in each equations are the unknowns, which will be solved by LPF. However, as not all factors impacting the performance can be measured, the linear equation system cannot be accurately solved. To allow for some error in the estimation of each benchmark, we introduce a Calibration Fudge factor $(\mathrm{CF})$. Finding the weights with the overall least absolute error $(\mathrm{CF})$ will yield the most accurate estimation. The LPF is as follows:

Minimise:

$\Sigma_{i}|\mathrm{CF}(\mathrm{i})|$

Subject to:

Benchmark $1: D_{11} \cdot W_{1}+D_{12} \cdot W_{2}+\ldots+D_{1 M} \cdot W_{M}+C F_{1}=\left(B_{1}\right)$

Benchmark2: $D_{21} \cdot W_{1}+D_{22} \cdot W_{2}+\ldots+D_{2 M} \cdot W_{M}+C F_{2}=\left(B_{2}\right)$

...

BenchmarkN : $D_{N 1} \cdot W_{1}+D_{N 2} \cdot W_{2}+\ldots+D_{N M} \cdot W_{M}+C F_{N}=\left(B_{N}\right)$

where $N$ is the number of benchmarks and $M$ is the number of operation type and data-type combinations. Each benchmark is represented by one equation. Adding benchmarks will increase information for the LP Solver to find the weights of that $\mathrm{PE}$, to then produce a more realistic weight table leading to a better future estimation. 


\section{Implementation}

This section presents the benchmarks, tools, PEs and metrics that are used for evaluating the approach.

Benchmarks used in the framework play an important role in estimation process. Balance in distribution of these benchmarks allows more accurate estimation for future applications. A major effort has been devoted to gather a suitable set of benchmarks that can cover most of operations, data types and coding structures. Table 1 shows the benchmarks. In Table 1, Randomly Gen-

Table 1. List and categories of benchmarks

\begin{tabular}{|c|c|}
\hline Source & Names of Benchmarks \\
\hline $\begin{array}{c}\text { MiBench [12] \& } \\
\text { DSP-Stone [13] }\end{array}$ & $\begin{array}{c}\text { AES whetstone bcnt blit bubblesort cnt crc crc2 edn fft1 fir2 } \\
\text { gamma hanoi heapsort linpack lms lms2 ludcmp matmult matrix } \\
\text { basicmath ndes nsichneu peakSpeed1 prime queens v42 wavelt }\end{array}$ \\
\hline $\begin{array}{c}\text { Randomly } \\
\text { Generated }\end{array}$ & $\begin{array}{c}\text { frand1 frand10 frand12 frand13 frand16 frand17 frand18 } \\
\text { frand19 frand2 frand20 frand3 frand4 frand6 frand8 } \\
\text { frand9 rand3 rand5 rand6 rand7 rand8 rand9 }\end{array}$ \\
\hline Synthetic & $\begin{array}{c}\text { synadddouble synaddfloat synaddlonglongint syndivdouble } \\
\text { syndivint syndivlonglongint synmindouble synminfloat synminint } \\
\text { synmulfloat synmulint synmullonglongint synmuldouble synaddint } \\
\text { synminlonglongint syndivfloat }\end{array}$ \\
\hline WCET & adpcm crc2 edn fft1 fir2 lms ndes nsichneu qurt \\
\hline
\end{tabular}

erated benchmarks have been generated using the modified Randprog tool [15]. Synthetic benchmarks predominately focus on a operation and data-type combination. They are only used for calibration only. The WCET benchmarks are a subset of those presented in [16].

We used the SCProf [1] profiler to extract application demand and gcc to compile the benchmarks for real hardware. We used the open source SCIP solver [17] to solve LPF. We have applied WeiCal to two different processors (Blackfin527 [14] and ARM9 [11]), with various hardware configurations (SRAM and SDRAM with Blackfin527 [14]) and compiler optimizations (O0, O1, O2, O3 with ARM9 [11]).

As metrics, we mostly use absolute error, comparing real execution cycles with estimated cycles. However, absolute accuracy may not always be required. During DSE, different design alternatives are compared. In this setting, the correctness of a relative comparison is sufficient. Fidelity quantifies the correctness of a relative comparison.

The dimensions of the weight table and specification characteristic table are defined by the number of possible operations (number of rows) and datatypes (number of columns). The SCProf profiler distinguishes 55 operations and 16 datatypes. This leads to 880 different pairs of operations over datatypes. In order to estimate 880 weights (execution delays), the LP formulation needs at least 880 training benchmarks. It is challenging to collect such a large number of suitable benchmarks. In addition, it would lengthen the LP solver run-time. To reduce the number of required benchmarks, we reduce the dimensionality of the 
weight table by grouping similar operations and datatypes based on architectural assumptions. Table 2 summarizes our assumptions. Overall, we group all operations into 12 different operation groups and all datatypes into 4 datatype groups. This dramatically reduces the of combinations from 880 down to 48 combinations of operations and datatypes.

Table 2. Grouping of operations and data types for dimensionality reduction

\begin{tabular}{|c|c|}
\hline Groups & Pristine entries in original weight table \\
\hline \hline constant & constant \\
\hline array access & array access, content of \\
\hline function call & function call, return \\
\hline post increment & post increment, pre increment, post decrement, pre decrement \\
\hline not & bitwise not, logical not \\
\hline multiply & multiply, multiply/assignment \\
\hline divide & divide, modulo, divide/assignment, modulo/assignment \\
\hline add & add, subtract, add/assignment, subtract/assignment \\
\hline branch & if, if else, for, while, do while, default, switch, case, break, continue \\
\hline shift left & shift right, shift left \\
\hline equal & all compare operations \\
\hline or & bitwise or, logical or, exclusive or, and, logical-and \\
\hline assignment & all possible assignment operations \\
\hline int & $\begin{array}{c}\text { unsigned long int, unsigned char, char, unsigned short, pointer, bool } \\
\text { short, unsigned int, long int, unsigned long }\end{array}$ \\
\hline long long int & long long int, unsigned long long int \\
\hline float & float, unsigned float \\
\hline double & double, long double, unsigned double \\
\hline
\end{tabular}

\section{Validation through Synthetic Model}

Any estimation approach is limited by the number of observable features (profiling restrictions), and by the complete availability of processor performance information (limited micro-architectural knowledge). To initially validate and optimise our approach under known conditions, we employ a synthetic model. We designed a statistical model which produces synthetic PEs and synthetic benchmarks to be used in WeiCal. Using synthetic model increases visibility over real measurements and processors. Each processor is modelled by a set of elements that contribute to delay in execution (such as execution of an operation, cache hit and cache miss). To mimic the effect of partial observability by the profiler, we declare some of these effects as observable, while other effects as non-observable. The number of training benchmarks and non-observable elements are varied to study their impact on the estimation accuracy. In order to realise the non-observable elements, their effect was deliberately included when calculating the measured execution time. However, the occurrence of nonobservable elements in benchmarks is hidden from the profiler. In result, the profiler counts only the observable elements, while the timing measurement includes delay (cycles) due to observable as well as non-observable elements. The 
LPF will attribute the effects of non-observable elements to the observable elements. As such, the number of cycles will increase for each observable feature. This is similar to other models which for example fold memory access delay statistically into operations.

Processor $P$ is modelled as

$$
P=\left[W_{k}\right]\left[W_{u}\right]
$$

where $\left[W_{k}\right]$ is the set of delays for known elements and $\left[W_{u}\right]$ is the set of delays of unknown elements. The particular values for $\left[W_{k}\right]$ and $\left[W_{u}\right]$ are randomly chosen (linear distribution) during the generation of a processor model.

Similarly, a synthetic benchmark is defined as recurrence of elements known and unknown to the Profiler.

$$
B=\left[R_{k}\right]\left[R_{u}\right]
$$

where $\left[R_{k}\right]$ is the set of recurrence for known elements and $\left[R_{u}\right]$ is the set of recurrence of unknown elements. The particular values for $\left[R_{k}\right]$ and $\left[R_{u}\right]$ are randomly chosen (linear distributed) during the generation of a benchmark.

The delay of a specific synthetic benchmark $i$ on a particular synthetic $\mathrm{PE} j$ is defined as

$$
(\text { Delay })_{i j}=\left[R_{k}\right]_{i}\left[W_{k}\right]_{j}+\left[R_{u}\right]_{i}\left[W_{u}\right]_{j}
$$

Figure 2 shows the effect of non-observable elements evaluated by changing the ratio of observable elements to non-observable elements in the synthetic model. The total number of elements was constant (60), while varying the nonobservable elements as 2\%,25\%,40\% and 60\%. The non-observable elements was set to contribute $15 \%$ of the total computation demand.

In the upper left graph of Figure 2, the mean estimation error quickly converges to $2 \%$ when the number of training benchmarks reaches 60 . With $25 \%$ non-observable elements, the mean estimation error stays at $12 \%$ with more than 60 training benchmarks (upper right). When non-observable elements increase to $40 \%$ or even $60 \%$, both the average and absolute estimation error are high. The estimation error is no longer improved with more than 60 training benchmarks.

It indicates that the number of training benchmarks need to be necessarily larger than the number of elements in order to converge the estimation error. However, even a large number of training benchmarks does not improve the accuracy of the weight table and corresponding estimation when the non-observable elements are more than $40 \%$. At least 50 training benchmarks are required for the estimation error to converge. The number of training benchmarks should be greater than the number of weights in the weight table for correct estimations.

The synthetic model allowed us to evaluate the effect of the non-observable elements and training benchmarks on the estimation error. The estimation accuracy improves with fewer non-observable elements. It is impossible to achieve a meaningful estimation with non-observable elements over $40 \%$ of all (observable and non-observable) architectural elements. Furthermore, the estimation error converges when the training benchmarks is slightly more than the desired observable elements. Adding more training benchmarks does not further improve the accuracy largely. 

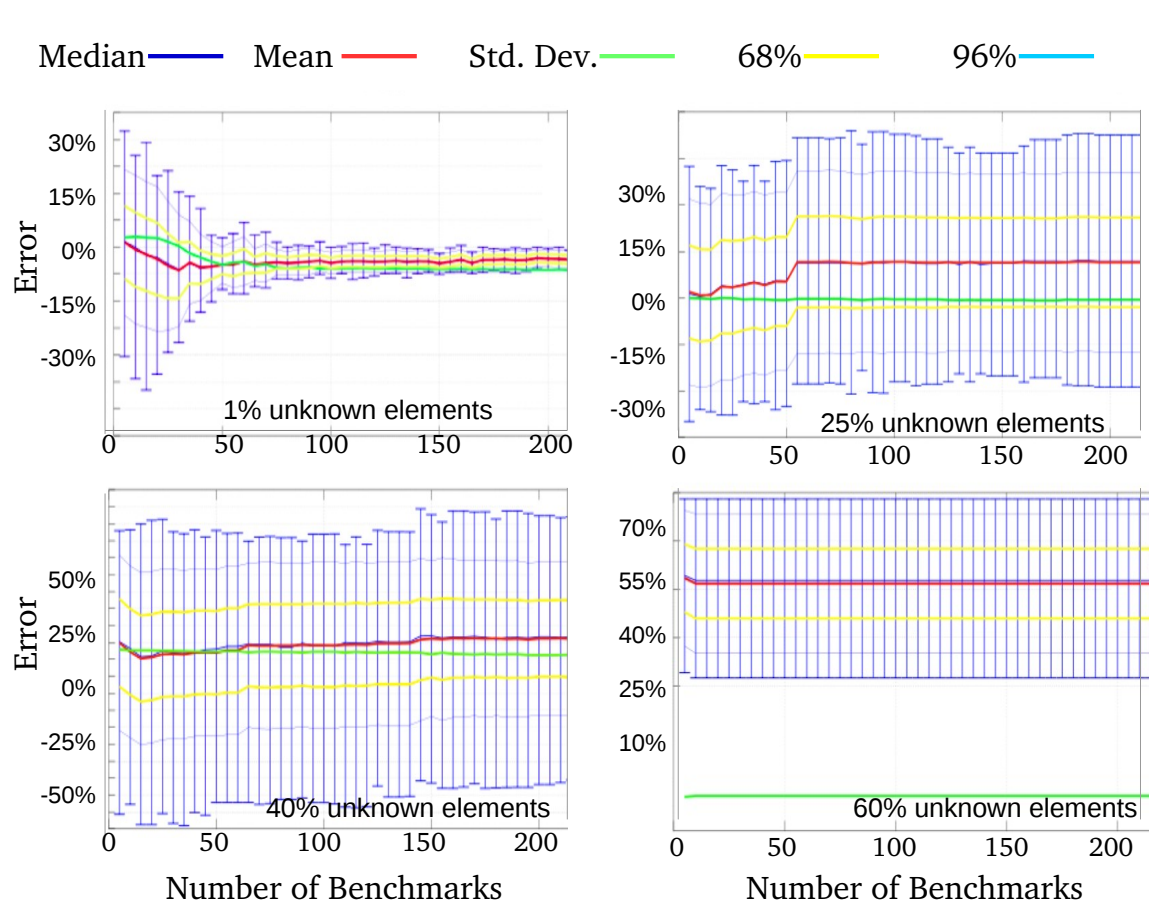

Fig. 2. Effect of number of benchmarks on average error

\section{Experimental Results}

The efficiency of our approach affects the high-level design decisions. On one hand, accuracy is desired to correctly guide the DSE. On the other hand, performance estimation should be fastest to evaluate many design combinations.

Accuracy is evaluated is terms of absolute error and fidelity. In order to measure estimation error, we excluded one benchmark from the training benchmarks and used it as a target application to determine estimation accuracy. The procedure was repeated through all the real benchmarks. Real and synthetic benchmarks were used for calibrating the weight tables. However, only the real benchmarks were used as test applications as they better reflect the characteristics of an actual workload. The results are aggregated in Figure 3.

Figure 3 shows the median, quartiles, minimum and maximum of the estimation errors among all benchmarks across all PEs. The box plot shows these static quantities, while the violin plots show the distribution of estimation error across the benchmarks. Figure 3 also shows the effect of compiler optimizations and hardware configurations on the estimation error. The majority of benchmarks have an estimation error close to median (-6\%) for Blackfin with SRAM. Moving from SRAM to SDRAM deteriorates the median to $-30 \%$. Most accurate results are achieved with low optimization. The ARM9 with O0 has the median at - $11 \%$ with most of the benchmarks having an estimation error close to median. As the optimization increases, the association of source code with the execution time reduces. This is due to the weak correlation between source-level $\mathrm{C}$ code and binary at higher optimization levels. With an increased optimization to $\mathrm{O} 1$ for 


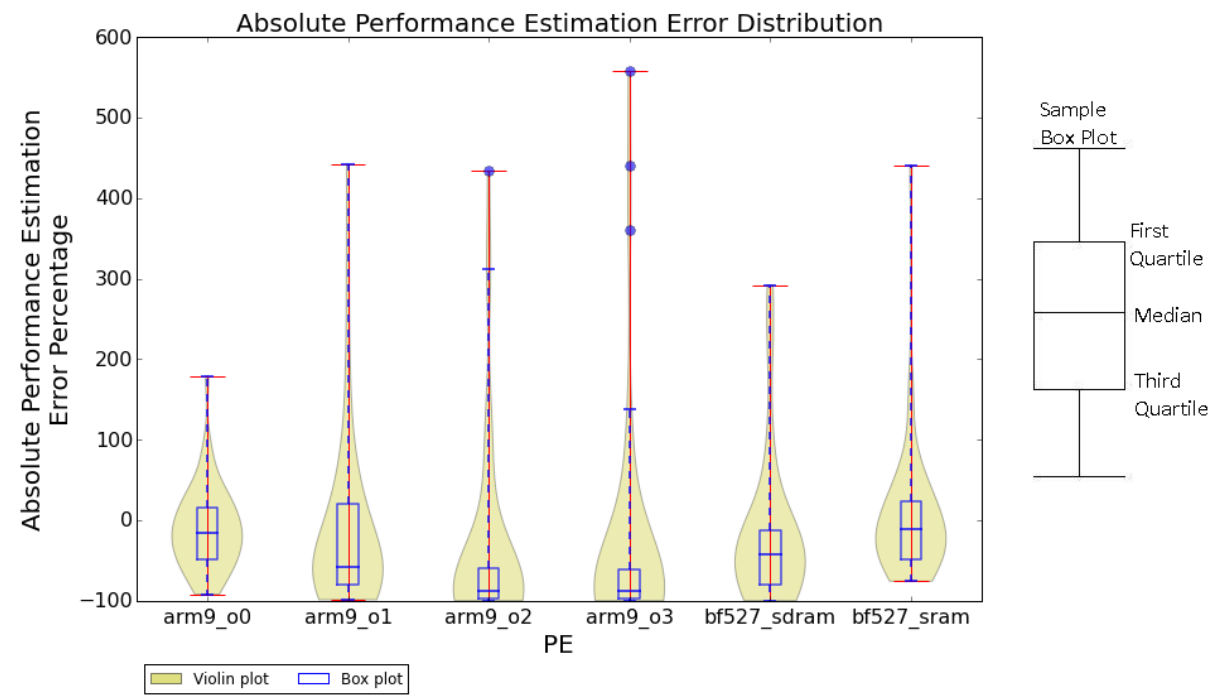

Fig. 3. Estimation Error on Real Platforms

ARM9, the median is at $-57 \%$. WeiCal is able to distinguish between various software and hardware configurations. Some applications do not perform well in this approach in terms of the estimation accuracy. This is the cost being paid for estimating at the highest level of abstraction. In some configurations, WeiCal has a higher absolute error than what was reported in [1] as we are now using more complex processors, memory hierarchies and compiler optimizations.

In early DSE, fidelity (i.e. relative comparison) is sufficient and absolute accuracy is less important. In order to analyse fidelity, we describe fidelity matrix, which shows the fidelity between all the possible pairs of PEs from the design space. Table 3 presents the fidelity matrix. To further analyse fidelity, we plot also fidelity over the measured performance gap between the investigated PE configurations. Fidelity depends on closeness between real performance of PEs being compared.

Table 3. Fidelity Matrix

\begin{tabular}{|c|c|c|c|c|c|c|}
\hline & BF527 SRAM & BF527 SDRAM & ARM9 O0 & ARM9 O1 & ARM9 O2 & ARM9 O3 \\
\hline BF527 SRAM & $100 \%$ & $90 \%$ & $89 \%$ & $76 \%$ & $80 \%$ & $80 \%$ \\
\hline BF527 SDRAM & - & $100 \%$ & $76 \%$ & $90 \%$ & $93 \%$ & $92 \%$ \\
\hline ARM9 O0 & - & - & $100 \%$ & $94 \%$ & $96 \%$ & $100 \%$ \\
\hline ARM9 O1 & - & - & - & $100 \%$ & $57 \%$ & $61 \%$ \\
\hline ARM9 O2 & - & - & - & - & $100 \%$ & $55 \%$ \\
\hline ARM9 O3 & - & - & - & - & - & $100 \%$ \\
\hline
\end{tabular}

Table 3 shows that performance estimation using calibrative weight tables has high fidelity with average fidelity being $82 \%$. Fidelity increases when performance gap between the compared PEs is larger. The estimated comparison of ARM9 O0 with its higher optimization counterparts is correct in more than $94 \%$ of the cases.

In addition to accuracy, the value of estimation methodology also depends on the time it takes for making an estimation. A separate aspect is the duration for generating a weight table (ie. performance of WeiCal). The weight table is determined only once in the lifetime of a PE and is less important. Hence, calibration takes place only once. We have automated executing benchmarks on target PEs 
and measuring execution duration (clock cycles). The average time it takes to load each benchmark is 2.5 seconds. The LPF took 0.6 seconds to generate the weight table for 48 given equations ( 48 benchmarks). The calibration phase took nearly 120 seconds in addition to the actual execution time of all benchmarks on the hardware.

Conversely to calibration, the estimation in retargeting to different PE configurations occurs very frequently during DSE. Thus, its performance is highly critical. Estimation is merely a matrix multiplication of the PE weight table and application profile table. As the dimensions of weight table and application profile table are fixed, the estimation time is independent of the application size and PE complexity. On a single core of 3.1 GHz i5-3450 Intel host, 25 million estimations / second can be obtained regardless of application size and PE complexity. Additionally, as the estimation is only a matrix multiplication, it's parallelization has been studied a lot. The average error of $24 \%$ is acceptable for early DSE where fidelity and high speed of estimation are more prominent factors.

\section{Conclusion}

Rapid estimation with sufficient fidelity is essential for DSE. In this context, retargetable source-level profiling [1] is a promising approach. It profiles a specification once to determine the specification computation demand. Then, estimating the application's execution time is as simple as a matrix multiplication of the specification computational demand and weight table capturing the PE's computation supply. However, this approach heavily relies on the quality and availability of weight tables.

The work presented in this paper proposes a calibration-based framework to automatically determine a processor's weight table(s). It avoids the manual and error-prone process of manual capturing processor characteristics (execution time). In particular, it mitigates the challenge of limited visibility of the sourcelevel profiling (i.e. C statements) and the associated challenge of attributing non-visible characteristics into the accounted operations.

We devised a synthetic model in order to validate the approach and analyse the bounds. We measured efficiency of the WeiCal using 49 benchmarks (mainly MiBench and DSP Stone) on ARM9 and Blackfin BF527 processors and considered memory configurations (SRAM and SDRAM) and software optimizations (O0, O1, O2 and O3). With the weight table approach 25 million estimations / second can be performed on a single core of $3.1 \mathrm{GHz}$ i5-3450 Intel host. The average estimation error was $24 \%$. However, the approach offers higher fidelity especially with larger performance gap between (e.g. above $94 \%$ fidelity for comparing ARM0 at O0 with other optimizations). The high estimation speed with

good fidelity makes this methodology an ideal cornerstone for an automated DSE.

\section{References}

1. Cai, Lukai and Gerstlauer, Andreas and Gajski, Daniel. Retargetable Profiling for Rapid, Early System-level Design Space Exploration. Proceedings of the 
41st Annual Design Automation Conference, DAC 2004, 1-58113-828-8, San Diego, CA, USA 281-286

2. Lattuada, M. and Ferrandi, F. Performance modeling of embedded applications with zero architectural knowledge. IEEE/ACM/IFIP International Conference on Hardware/Software Codesign and System Synthesis (CODES+ISSS), 2010. 277-286.

3. Zhonglei Wang and Herkersdorf, A. An efficient approach for system-level timing simulation of compiler-optimized embedded software. 46th ACM/IEEE, Design Automation Conference, 2009, 220-225.

4. Yan Lin Aung and Siew-Kei Lam and Srikanthan, T. Compiler-assisted technique for rapid performance estimation of FPGA-based processors. IEEE International SOC Conference (SOCC), 2011, 341-346.

5. Oyamada, M. and Wagner, F. R. and Bonaciu, M. and Cesario, W. and Jerraya, A. Software Performance Estimation in MPSoC Design. Asia and South Pacific Design Automation Conference Proceedings of the 2007, 1-4244-0629-3, 38-43.

6. Gao, L. and Karuri, K. and Kraemer, S. and Leupers, R. and Ascheid, G. and Meyr, H. Multiprocessor performance estimation using hybrid simulation. 45th ACM/IEEE Design Automation Conference, 2008, 325-330.

7. Yonghyun Hwang and Abdi, S. and Gajski, D. Cycle-approximate Retargetable Performance Estimation at the Transaction Level. Design, Automation and Test in Europe, 2008. DATE '08, 3-8.

8. Javaid, H. and Janapsatya, A. and Haque, M.S. and Parameswaran, S. Rapid runtime estimation methods for pipelined MPSoCs. Design, Automation Test in Europe Conference Exhibition, 2010, 363-368.

9. Stattelmann, S. and Bringmann, O. and Rosenstiel, W. Fast and accurate source-level simulation of software timing considering complex code optimizations. Design Automation Conference (DAC), 2011 48th ACM/EDAC/IEEE, 2011, 486-491.

10. Mohanty, S. and Prasanna, V.K. Rapid system-level performance evaluation and optimization for application mapping onto SoC architectures. 15th Annual IEEE International ASIC/SOC Conference, 2002, 160-167.

11. Samsung Electronics. 32 bit CMOS Microcontroller User's Manual. S3C2440A, Jul, 2004, Rev 1.

12. Matthew Guthaus and Jeffrey Ringenberg and Dan Ernst and Todd Austin and Trevor Mudge and Richard Brown. MiBench: A Free, Commercially Representative Embedded Benchmark Suite. IEEE International Workshop on Workload Characterization WWC-4, Dec, 2001, 3-14.

13. V. Zivojnovic and J. Martinez and C. Schlager and H. Meyr. DSPstone: A DSP-Oriented Benchmarking Methodology, The International Conference on Signal Processing Applications and Technology, 1994, 715-720.

14. Analog Devices. Blackfin Embedded Processor. ADSP-BF527, 2013, Rev. D.

15. Eric Eide and John Regehr. Volatiles Are Miscompiled, and What to Do about It. EMSOFT, 2008.

16. Jan Gustafsson and Adam Betts and Andreas Ermedahl and Bjorn Lisper. The Malardalen WCET Benchmarks - Past, Present and Future. WCET2010, 137-147, 2010, Brussels, Belgium.

17. Achterberg, T. Mathematical Programming Computation. SCIP: Solving Constraint Integer Programs, 2009, Jul, 1-41. 